\title{
Review Article \\ Roles of Nicotinamide N-Methyltransferase in Obesity and Type 2 Diabetes
}

\author{
Jie-Ru Liu, Zhao-Hui Deng, Xiao-Juan Zhu, Yu-Rong Zeng, Xiang-Xiang Guan, \\ and Jiang-Hua Li
}

\begin{abstract}
Key Lab of Aquatic Training Monitoring and Intervention of General Administration of Sport of China, Physical Education College, Jiangxi Normal University, Nanchang, 330022 Jiangxi, China
\end{abstract}

Correspondence should be addressed to Jiang-Hua Li; lijianghua8@sina.com

Received 8 March 2021; Accepted 20 July 2021; Published 28 July 2021

Academic Editor: Sheba Mohankumar

Copyright (c) 2021 Jie-Ru Liu et al. This is an open access article distributed under the Creative Commons Attribution License, which permits unrestricted use, distribution, and reproduction in any medium, provided the original work is properly cited.

\begin{abstract}
Type 2 diabetes (T2D) is thought to be a complication of metabolic syndrome caused by disorders of energy utilization and storage and characterized by insulin resistance or deficiency of insulin secretion. Though the mechanism linking obesity to the development of T2D is complex and unintelligible, it is known that abnormal lipid metabolism and adipose tissue accumulation possibly play important roles in this process. Recently, nicotinamide N-methyltransferase (NNMT) has been emerging as a new mechanism-of-action target in treating obesity and associated T2D. Evidence has shown that NNMT is associated with obesity and T2D. NNMT inhibition or NNMT knockdown significantly increases energy expenditure, reduces body weight and white adipose mass, improves insulin sensitivity, and normalizes glucose tolerance and fasting blood glucose levels. Additionally, trials of oligonucleotide therapeutics and experiments with some small-molecule NNMT inhibitors in vitro and in preclinical animal models have validated NNMT as a promising therapeutic target to prevent or treat obesity and associated T2D. However, the exact mechanisms underlying these phenomena are not yet fully understood and clinical trials targeting NNMT have not been reported until now. Therefore, more researches are necessary to reveal the acting mechanism of NNMT in obesity and T2D and to develop therapeutics targeting NNMT.
\end{abstract}

\section{Introduction}

Obesity is a predisposing factor for $\mathrm{T} 2 \mathrm{D}$, and the prevalence of obesity has significantly accelerated the rise of T2D in the past few decades. T2D is thought to be a complication of metabolic syndrome caused by disorders of energy utilization and storage [1] and characterized by insulin resistance and deficiency of insulin secretion [2]. The mechanism linking obesity and the development of T2D is complex and unintelligible, but it is known that abnormal lipid metabolism and adipose tissue accumulation possibly play important roles [3]. The increase in the circulatory free fatty acid (FFA) concentration in plasma and the elevation of lipid deposition in skeletal muscles caused by abnormal lipid metabolism are the important signs of insulin resistance and T2D [4]. Increased plasma FFAs reduce glucose uptake stimulated by insulin, but the decrease of lipid content improves insulin activities in skeletal muscles, adipocytes, and the livers [4, 5]. It was proposed that the elevated levels of FFAs in plasma causes insulin resistance by inhibiting glucose transport and/or phosphorylation activities [4]. Adipose tissues release numerous metabolic hormones, cytokines, and other mediators, such as glycerol, leptin, adiponectin, and nonesterified fatty acids (NEFAs) [6]. An increase in the NEFA level in plasma is observed in patients with obesity and T2D and is significantly associated with insulin resistance [6, 7]. Increased NEFAs in cells compete with glucose for substrate oxidation, thus resulting in the inhibition of pyruvate dehydrogenase, phosphofructokinase, and hexokinase II [8].

Although it is known that abnormal lipid metabolism and adipose tissue accumulation possibly play important roles in linking obesity and $\mathrm{T} 2 \mathrm{D}$, the actual mechanisms are still unclear because not all obese people are insulin resistant or at high risk of diabetes [9]. Recently, NNMT has been 
emerging as a new mechanism-of-action target for treatment of obesity and associated T2D [10]. This review will focus on the roles of NNMT in obesity and T2D, as it has been found that the expression of this methyltransferase is elevated in the white adipose tissue and liver of obese people and diabetic mice and that NNMT knockdown protects against dietinduced obesity and insulin resistance [11].

\section{Physiological Function of NNMT and the Related Metabolic Pathways}

NNMT is a cytosolic enzyme that catalyzes NA methylation using S-adenosyl-methionine (SAM) as a methyl donor, while producing 1-methylnicotinamide (MNA) and Sadenosyl-homocysteine (SAH). As Figure 1 shows, NA is a precursor of $\mathrm{NAD}^{+}$and also a catabolic product of $\mathrm{NAD}^{+}$. $\mathrm{NAD}^{+}$is mainly consumed in three ways, in which NA is produced, and 4 enzymes, sirtuins, ADP-ribosyl transferases (ARTs), poly-ADP-polymerases (PARPs), and cADP-ribose synthases, are involved (Figure 1). Therefore, the methylation of NA is not only relevant to the abundance of free $\mathrm{NAD}^{+}$but is also relevant to the activity of these $\mathrm{NAD}^{+}$-consuming enzymes, because as a metabolite of these $\mathrm{NAD}^{+}$consuming reactions, NA naturally has inhibitive effects on these enzymes.

$\mathrm{NAD}^{+}$is the key coenzyme for glycolysis and the tricarboxylic acid cycle and for the conversion of carbohydrates to lipids [12]. Sirtuins are a family of $\mathrm{NAD}^{+}$-dependent deacylases that have been shown to regulate a myriad of biological functions, ranging from cell growth to lifespan extension, including oxidative stress, DNA damage, glycolysis, gluconeogenesis, and lipogenesis [13-16]. ARTs, PARPs, and cADP-ribose synthases cleave $\mathrm{NAD}^{+}$into NA and ADPribose (Figure 1). ADP-ribose is polymerized onto the nuclear proteins, including histones and transcription factors at DNA strand breaks, in DNA repair [17]. Normal concentrations of NA can prevent degradation of PARPs and allow DNA repair [18]. NA cannot be salvaged into $\mathrm{NAD}^{+}$any longer if it is N-methylated by NNMT, so the methylation of NA impairs the abundance of free $\mathrm{NAD}^{+}$and then affects the activities of these $\mathrm{NAD}^{+}$-consuming enzymes [19]. According to these findings, it was proposed that NNMT plays important roles in energy metabolism and in the development of a number of disorders, such as obesity, diabetes, aging, Parkinson's disease, and cancer [19]. This review article is based on works with a focus on the current research status quo of NNMT in the field of obesity and T2D.

\section{Association between NNMT and Obesity}

Early evidence of the association between NNMT and obesity was observed in metabolomic works, of which the results show that the MNA levels in human urine are positively correlated with the body mass index (BMI) [20,21]. Recently, this association has been confirmed by many reports. For instance, the MNA levels in serum were found increased in the obese people [22] and the MNA levels in urine were found increased both in obese $\mathrm{db} / \mathrm{db}$ mice and in obese Zucker rats [21]. These findings indicate that the activity of
NNMT is increased in obese individuals. Evidence has shown that NNMT expression in white adipose tissue (WAT) is high in obesity-prone mice and low in obesity-resistant mice [23-25] and significantly correlates with the percent fat mass in diet-induced obese mice [26]. Lee et al. found that NNMT expression in adipocytes is increased in Pima Indians [27]. Kraus et al. [11] found that NNMT knockdown induced a $47 \%$ reduction in the relative fat mass of mice and proposed that NNMT in WATs and/or in livers may have a causative role in obesity. Brachs et al. [28] found that NNMT knockdown significantly reduced the body weight, fat mass, and insulin level of female mice fed a Western diet (fat $47 \% \mathrm{kcal}$, carbohydrate $34 \% \mathrm{kcal}$ ), as did NNMT knockout.

Since NNMT expression is directly determined by the NNMT gene, in our previous works, nineteen SNPs in the NNMT DNA sequence were selected as tagSNPs using Haploview software (Haploview 4.2) to observe the association between NNMT gene polymorphisms and obesity [29] and hyperlipidemia [30]. We found that the rs10891644 variation is significantly associated with obesity [29] and that the rs1941404 variation is significantly associated with hyperlipidemia in the Chinese population [30]. Recently, Bañales-Luna et al. [31] reported that Mexican subjects with rs694539 variation (genotype AA, recessive inherence model) in the NNMT gene sequence have lower BMI values.

\section{Association between NNMT and T2D}

Many reports have shown the association between NNMT and T2D. Quantitative trait locus mapping in mice demonstrated that NNMT plays a causative role in the development of T2D [32] and MNA levels were elevated in both the serum [22] and urine [21] of patients with T2D. These results indicate that NNMT activities are increased in the development of T2D. The more direct evidence is mainly from the following reports. Kannt et al. [33] demonstrated that NNMT expression is upregulated in WAT of humans with insulin resistance or T2D and that plasma MNA levels are significantly and positively correlated with NNMT expression in WAT and with the degree of insulin resistance. Kraus et al. [11] found that NNMT expression levels are elevated in WAT and the liver of the T2D mice and that NNMT knockdown improves the glucose tolerance and insulin sensitivity in the T2D mice. Subsequently, Hong et al. [14] confirmed the roles of NNMT in the development of T2D. In vitro, they found that NNMT knockdown significantly lowers hepatocyte glucose output (50\%) and that NNMT overexpression significantly increases hepatocyte glucose output (1.4-fold) [14]. In vivo, they found that NNMT knockdown in the livers of C57BL6/J mice significantly lowers overnight fasting glucose levels [14].

In our previous works, the association between T2D and two SNPs in the NNMT gene sequence (rs694539 and rs1941404) was observed [34]. The rs694539 variation has shown the significant associations with many noninfectious chronic diseases (NCDs), such as hyperhomocysteinemia [35], nonalcoholic steatohepatitis [36], congenital heart diseases [37], migraine [38], bipolar disorder [39], schizophrenia [40], epilepsy [41], and abdominal aortic diseases 


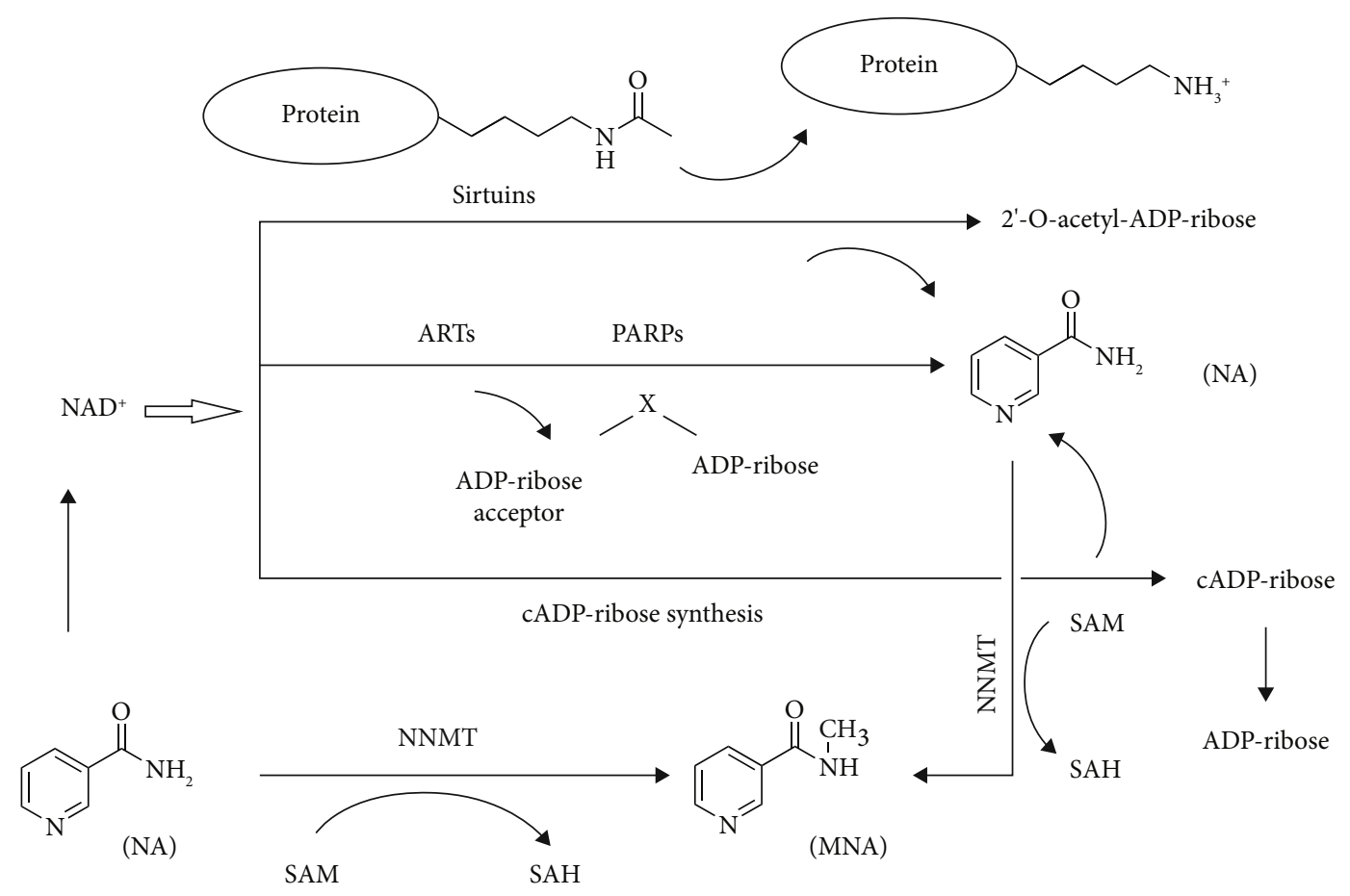

Figure 1: Physiological function of nicotinamide N-methyltransferase (NNMT) and the related metabolic pathways. NA: nicotinamide; MNA: 1-methylnicotinamide; SAM: S-adenosyl-methionine; SAH: S-adenosyl-homocysteine; $\mathrm{NAD}^{+}$: nicotinamide adenine dinucleotide; ARTs: ADP-ribosyl transferases; PARPs: poly-ADP-polymerases.

[42], and the rs1941404 variation has been reported to be associated with hyperlipidemia [30]. The results show that the rs1941404 variation is significantly associated with T2D [34].

\section{Potential Mechanisms Linking NNMT and T2D}

T2D is thought to be a complication of metabolic syndrome caused by an underlying disorder of energy metabolism and characterized by impaired glucose tolerance or elevated fasting blood glucose [43]. In this section, we will focus on the roles of NNMT in regulating energy metabolism and glucose metabolism.

5.1. Roles of NNMT in Energy Metabolism and the Related Mechanisms. As a precursor of $\mathrm{NAD}^{+}$, NA methylation plays important roles in regulating energy metabolism because methylated NA is no longer able to be salvaged into $\mathrm{NAD}^{+}[16] . \mathrm{NAD}^{+}$is a key coenzyme for fuel oxidation and for the conversion of carbohydrates to lipids [44]. The competition between NA methylation and $\mathrm{NAD}^{+}$salvage indicates that NNMT might limit fuel oxidation and promote fat storage, because if the activity or expression of NNMT is high, then, NA might not be salvageable, thus limiting the $\mathrm{NAD}^{+}$-dependent processes [16].

In our previous works, we found that NNMT genetic variants in the Chinese population are significantly associated with resting energy expenditure [30] and maximal oxygen uptake [45]. Recently, Bañales-Luna et al. [31] reported that NNMT genetic variants in Mexican subjects are also significantly associated with resting energy expenditure. The further roles of NNMT in regulating energy metabolism and the related mechanism were mainly from Kraus et al. [11]. In vitro, they found that NNMT knockdown and NNMT inhibition by MNA both significantly increase the oxygen consumption in adipocytes. However, NNMT overexpression significantly decreases the oxygen consumption in adipocytes [11]. In vivo, they found that NNMT knockdown mice had higher energy expenditures than the control mice with similar body weights [11]. Regarding the mechanism by which NNMT regulates energy expenditure, they proposed that SAM and $\mathrm{NAD}^{+}$ are involved because SAM and $\mathrm{NAD}^{+}$are modulated in the process of NA methylation by NNMT [11]. $\mathrm{NAD}^{+}$is a key cofactor linking cellular redox states to energy metabolism [46]. SAM provides propylamine for the biosynthesis of polyamine and donates a methyl group for the methylation of histone [47]. Activation of polyamine flux causes an increase in energy expenditure by catalyzing polyamine acetylation to produce acetylpolyamines using acetyl-coA as a substrate [48]. NNMT inhibition increases the levels of SAM and $\mathrm{NAD}^{+}$and upregulates the activation of polyamine flux, resulting in an increase in energy expenditure [11].

5.2. Roles of NNMT in Glucose Metabolism and the Related Mechanisms. As mentioned above, T2D is characterized by impaired glucose tolerance or elevated fasting blood glucose [43]. In our previous works, a SNP (rs1941404) in the NNMT gene sequence was found significantly associated with impaired glucose tolerance and elevated fasting blood glucose [34]. The further roles of NNMT in regulating glucose 
metabolism and the related mechanisms are mainly from Kraus et al. [11] and Hong et al. [14].

Kraus et al. [11] reported that NNMT knockdown improves glucose tolerance and insulin sensitivity in dietinduced obese mice. Brachs et al. [28] confirmed these findings in their studies. They found that NNMT knockdown reduced the insulin level and improved glucose tolerance in female mice fed with a Western diet and that NNMT knockout strongly improved insulin sensitivity in male mice fed with a high-fat diet [28]. Hong et al. [14] initially tested the roles of NNMT in gluconeogenesis and found that NNMT knockdown decreases hepatocyte glucose output, glucose-6-phosphatase catalytic (G6pc) expression, and phosphoenolpyruvate carboxykinase 1 cytosolic (Pck1) expression in primary hepatocytes. On the contrary, NNMT overexpression increases hepatocyte glucose output, G6pc expression, and Pck1 expression in primary hepatocytes. In vivo, Hong et al. [14] found that mice with NNMT knockdown have lower levels of fasting glucose and lower pyruvate conversion to glucose compared to control mice. These findings suggest that NNMT is a positive modulator of hepatocyte gluconeogenesis.

Regarding the mechanism by which NNMT regulates glucose metabolism, Hong et al. [14] proposed that the regulatory effect of NNMT is mediated by its product MNA and that Sirt1 is necessary in this regulatory process. First, they found that glucose production, G6pc expression, and Pck1 expression were significantly lower in NNMT-overexpressing hepatocytes with Sirt1 inhibition than in NNMT-overexpressing hepatocytes without Sirt1 inhibition and that Sirt1 overexpression rescued the suppression of G6pc expression and Pckl expression induced by NNMT knockdown [14]. These results indicate that Sirt1 is necessary to mediate the regulatory effect of NNMT on glucose metabolism. Second, they found that Sirt1 protein expression is significantly correlated with NNMT expression in hepatocytes [14]. In vitro, Sirt1 protein expression was significantly increased in primary hepatocytes by NNMT overexpression and was significantly decreased in primary hepatocytes by NNMT knockdown [14]. In vivo, Sirtl protein expression was also significantly decreased in the livers of mice by NNMT knockdown [14]. Third, they found that the enzymatic activity of NNMT is necessary for the increase in Sirtl protein expression and a product of NNMT (MNA) plays a key role [14]. Similar to NNMT overexpression, MNA-treated hepatocytes show a dose-dependent increase in glucose production, Sirt1 protein expression, G6pc mRNA expression, and Pck1 mRNA expression compared to controls and these changes caused by MNA treatment can be abolished by Sirt1 knockdown [14]. These findings suggest that both NNMT and its product MNA increase Sirt1 protein expression, while Sirt1 is needed for the regulatory effects of NNMT and MNA on glucose metabolism [14].

\section{NNMT as a Therapeutic Target}

Evidence has shown that NNMT knockdown leads to an increase in energy expenditure in adipocytes [11] and a decrease in glucose output in hepatocytes [14]. Moreover, in animals, NNMT knockdown improves insulin sensitivity, overnight fasting glucose levels, and glucose tolerance and reduces the relative fat mass $[11,14]$. These findings render NNMT an attractive target in developing oligonucleotide drugs to prevent or treat obesity and T2D. However, to date, oligonucleotide therapeutics are still facing some technological obstacles, such as how to achieve efficient oligonucleotide delivery to target organs or tissues, how to overcome off-target interactions [49-52], and how to address the sequence- and chemistry-dependent toxicity and saturation of endogenous RNA processing pathways $[53,54]$. Therefore, although some oligonucleotide products targeting NNMT have shown effectiveness in vitro and in animal experiments, to date, no oligonucleotide products targeting NNMT have been used in clinical trials. However, it is noteworthy that ten oligonucleotide drugs have been officially approved by FDA as of January 2020 [55], which means that the technological obstacles for oligonucleotide therapeutics are being solved step by step and the development of oligonucleotide drugs targeting NNMT is still a promising method for the prevention or treatment of obesity and related $\mathrm{T} 2 \mathrm{D}$ in the future.

In addition to oligonucleotide therapeutics, some smallmolecule NNMT inhibitors have been reported recently [56-59] and several have shown effectiveness in the prevention or treatment of obesity and T2D in vitro $[60,61]$ or in preclinical animal models $[10,58,62]$, validating NNMT as a pharmacological drug target.

MNA, as the product of the NNMT enzymatic reaction, is widely used as an NNMT inhibitor in research on biological function of NNMT. In vitro, MNA has shown significant effects in increasing energy expenditure and modulating glucose metabolism [11, 14], but in vivo, its effects are controversial. In a report from Przyborowski et al. [63], after 4 weeks of MNA administration $(100 \mathrm{mg} / \mathrm{kg})$, no significant differences were found between MNA-treated and untreated diabetic $\mathrm{db} / \mathrm{db}$ mice with regard to the levels of fasting blood glucose and HbAlc and the body weights of the MNAtreated $\mathrm{db} / \mathrm{db}$ mice did not decrease but tended to increase compared to those of the untreated $\mathrm{db} / \mathrm{db}$ mice. Hong et al. [14] also reported that MNA supplementation has no effects on blocking body weight gain. As a product of the NNMT enzymatic reaction, MNA is a natural inhibitor of NNMT. Swaminathan et al. [64] demonstrated the ternary complex X-ray crystal structures of NNMT in bound form with MNA, which indicates that MNA can bind to the active site of NNMT, thereby inhibiting NA binding and inhibiting the activities of NNMT. Why is the effect of MNA treatment in vivo not as good as it should be in the control of fasting blood glucose, $\mathrm{HbAlc}$, and body weight? There are 2 factors that might explain this phenomenon. First, MNA is not stable in in vivo. It can be further oxidized to N-methyl-2pyridone-5-carboxamide (2PY) and N-methyl-4-pyridone3 -carboxamide (4PY) in vivo and is easily excreted in urine in the forms of MNA, 2PY, and 4PY (in humans, the major metabolite of MNA is $2 \mathrm{PY}$, and in rodents, the major metabolite of MNA is 4PY) [65-67]. Second, the membrane permeability of MNA is poor. Membrane permeability is crucial for a drug to obtain desirable treatment effects. 
According to the report from Neelakantan et al. [10], MNA exhibited no passive permeability in parallel artificial membrane permeability assay (PAMPA), which might partly explain the undesirable treatment effects of MNA in the control of body weight and blood glucose homeostasis.

Neelakantan et al. [10] found some 1-methylquinolinium scaffolds modified with primary amine substitutions displaying both high passive membrane diffusion in PAMPA and high active transport membrane permeability in a bidirectional permeability assay with Caco- 2 cells, and one of them (5-amino-1-methylquinolinium (5-amino-1MQ)) has significant effectiveness against diet-induced obesity. Importantly, 5-amino-1MQ has high selectivity and does not inhibit the related SAM-dependent methyltransferases or enzymes in $\mathrm{NAD}^{+}$salvage pathways. In vitro, 5-amino-1MQ significantly reduced intracellular $\mathrm{MNA}$, increased intracellular $\mathrm{NAD}^{+}$, and suppressed lipogenesis in adipocytes. In vivo, 5amino-1MQ-treated mice had significantly reduced body weights, white adipose masses, and adipocyte sizes. Notably, 5-amino-1MQ treatment did not show a significant impact on the food intake and any observable adverse effects. These results suggest that 5-amino-1MQ is a potent small-molecule NNMT inhibitor that reverses diet-induced obesity and related $\mathrm{T} 2 \mathrm{D}$.

In addition to 5 -amino-1MQ, JBSNF-000088 is another NNMT inhibitor that is promising for treatment of obesity and associated T2D. JBSNF-000088, a small-molecule analog of NA, is able to be bound to NNMT and to be methylated in the NNMT structure, with SAM being correspondingly demethylated to SAH [58]. Therefore, the methylation of NA catalyzed by NNMT is inhibited, and the product, MNA, is reduced. In animal models of metabolic disease, JBSNF-000088 treatment reduced MNA levels and drove insulin sensitization, glucose modulation, and body weight reduction. In diet-induced obese mice, JBSNF-000088 treatment reduced body weights, improved insulin sensitivity and normalized glucose tolerance [58].

\section{Implications and Recommendations for Future Studies}

As summarized in this review, NNMT plays important roles in obesity and T2D and is an attractive therapeutic target to prevent or treat obesity and associated T2D. Although the current evidence is very promising, there are still some knowledge gaps that should be addressed to further clarify the exact mechanisms linking NNMT to obesity and T2D and to develop clinical drugs targeting NNMT.

Usually, increased energy expenditure is used to explain the protective effects of NNMT inhibition or NNMT knockdown against diet-induced obesity. NNMT catalyzes the methylation of NA, which is a precursor of $\mathrm{NAD}^{+}$. If NNMT expression or activity is high, then, NA might not be salvageable to form $\mathrm{NAD}^{+}$. The competition between NA methylation and $\mathrm{NAD}^{+}$salvage indicates that NNMT might limit fuel oxidation and promote fat storage because $\mathrm{NAD}^{+}$is necessary for fuel oxidation [16]. Kraus et al. [11] observed that NNMT knockdown increased both $\mathrm{NAD}^{+}$levels and oxygen consumption in WAT. However, in the liver, NNMT overexpression did not change the levels of $\operatorname{NAD}^{+}[11,14]$ but did depress oxygen consumption [11]. Therefore, the depression of energy expenditure caused by NNMT overexpression cannot be explained solely by the competition between NNMT and $\mathrm{NAD}^{+}$salvaging. In addition, it seems that NNMT plays different roles in aerobic and anaerobic energy metabolism. If the depressed oxygen consumption caused by NNMT overexpression indicates the depression of aerobic metabolism, the regulatory effect of NNMT on anaerobic metabolism might be reversed. Generally, NNMT activity or expression is significantly elevated in cancers, such as brain [68], lung [69], liver [70], kidney [71, 72], bladder [73], stomach [74, 75], pancreas [76], colon [77], and oral $[78,79]$ cancer but cancer cells usually have a significantly higher anaerobic metabolism rate than normal cells. In one of our previous studies [80], we found that the levels of NNMT expression in extensor digitorum longus muscles (a typical type of fast-twitch muscle relying on anaerobic metabolism to produce power) are higher than those in the soleus muscle (a typical type of slow-twitch muscle relying on aerobic metabolism to produce power) and that anaerobic exercise is more effective in increasing NNMT expression in skeletal muscles compared to aerobic exercise. Recently, we inhibited NNMT in rats with MNA and found that NNMT inhibition impaired performance of the rats in anaerobic endurance exercise. These phenomena indicate that NNMT might promote anaerobic metabolism [81]. Therefore, more studies are needed in the future to further clarify the roles of NNMT in energy metabolism and the related mechanisms.

NNMT inhibition or NNMT knockdown improves insulin sensitivity and normalizes glucose tolerance and fasting blood glucose levels [14], but the mechanisms by which NNMT regulates insulin sensitivity and blood glucose homeostasis are unclear. The liver is the major organ that maintains blood glucose homeostasis in mammals. When blood glucose levels are high, more insulin is excreted from the pancreas, and then, hepatocytes uptake more glucose from the blood to form glycogen under the action of insulin. When blood glucose levels are low, more glucose is produced in the liver by glycogenolysis and gluconeogenesis and then released into the blood. The liver is the organ with the highest level of NNMT expression in mammals. Hong et al. [14] demonstrated that NNMT is a positive modulator in hepatocyte gluconeogenesis but the roles of NNMT in glycogen metabolism in the liver and the mechanisms by which NNMT affects insulin sensitivity have not yet been reported.

\section{Conclusion}

Evidence has shown that NNMT plays important roles in obesity and T2D and that NNMT inhibition significantly increases energy expenditure, reduces body weight and white adipose mass, improves insulin sensitivity, and normalizes glucose tolerance and fasting blood glucose levels. However, the exact mechanisms underlying these phenomena are not yet fully understood. Additionally, trials of oligonucleotide therapeutics and some small-molecule NNMT inhibitors in vitro and in preclinical animal models have validated NNMT as a promising therapeutic target to prevent or treat 
obesity and related T2D, but currently, clinical trials have not yet been reported. Therefore, more researches are still necessary to reveal the roles and action mechanisms of NNMT in obesity and T2D and to develop therapeutics targeting NNMT.

\section{Conflicts of Interest}

There are no potential conflicts of interest.

\section{Authors' Contributions}

Liu JR, Deng ZH, Zhu XJ, Zeng YR, Guan XX, and Li JH contributed to this paper.

\section{Acknowledgments}

This study is supported by the National Natural Science Foundation of China (NSFC 21365013) and the Key Lab of Aquatic Training Monitoring and Intervention of General Administration of Sport of China (no. 201901).

\section{References}

[1] J. D. Mul, K. I. Stanford, M. F. Hirshman, and L. J. Goodyear, "Exercise and regulation of carbohydrate metabolism," Progress in molecular biology and translational science, vol. 135, pp. 17-37, 2015.

[2] American Diabetes Association, "Diagnosis and classification of diabetes mellitus," Diabetes Care, vol. 36, Suppl 1, pp. S67-S74, 2013.

[3] A. Pandey, S. Chawla, and P. Guchhait, "Type-2 diabetes: current understanding and future perspectives," IUBMB Life, vol. 67, no. 7, pp. 506-513, 2015.

[4] G. Boden, "Role of fatty acids in the pathogenesis of insulin resistance and NIDDM," Diabetes, vol. 46, no. 1, pp. 3-10, 1997, Erratum in: Diabetes 1997 Mar; 46(3): 536.

[5] J. D. McGarry, "Banting lecture 2001: dysregulation of fatty acid metabolism in the etiology of type 2 diabetes," Diabetes, vol. 51, no. 1, pp. 7-18, 2002.

[6] K. E. Wellen and G. S. Hotamisligil, "Inflammation, stress, and diabetes," The Journal of clinical investigation, vol. 115, no. 5, pp. 1111-1119, 2005.

[7] S. E. Shoelson, J. Lee, and A. B. Goldfine, "Inflammation and insulin resistance," The Journal of clinical investigation, vol. 116, no. 7, pp. 1793-1801, 2006, Erratum in: J Clin Invest. 2006 Aug; 116(8): 2308.

[8] P. J. Randle, P. B. Garland, C. N. Hales, and E. A. Newsholme, "The glucose fatty-acid cycle. Its role in insulin sensitivity and the metabolic disturbances of diabetes mellitus," Lancet, vol. 1, no. 7285, pp. 785-789, 1963.

[9] J. E. Hall, E. D. Crook, D. W. Jones, M. R. Wofford, and P. M. Dubbert, "Mechanisms of obesity-associated cardiovascular and renal disease," The American Journal of the Medical Sciences, vol. 324, no. 3, pp. 127-137, 2002.

[10] H. Neelakantan, V. Vance, M. D. Wetzel et al., "Selective and membrane-permeable small molecule inhibitors of nicotinamide $\mathrm{N}$-methyltransferase reverse high fat diet-induced obesity in mice," Biochemical pharmacology, vol. 147, pp. 141-152, 2018.
[11] D. Kraus, Q. Yang, D. Kong et al., "Nicotinamide Nmethyltransferase knockdown protects against diet-induced obesity," Nature, vol. 508, no. 7495, pp. 258-262, 2014.

[12] A. A. Sauve, "NAD ${ }^{+}$and vitamin B3: from metabolism to therapies," Journal of Pharmacology and Experimental Therapeutics, vol. 324, no. 3, pp. 883-893, 2008.

[13] M. C. Haigis and L. P. Guarente, "Mammalian sirtuins-emerging roles in physiology, aging, and calorie restriction," Genes \& Development, vol. 20, no. 21, pp. 2913-2921, 2006.

[14] S. Hong, J. M. Moreno-Navarrete, X. Wei et al., "Nicotinamide _N_-methyltransferase regulates hepatic nutrient metabolism through Sirtl protein stabilization," Nature medicine, vol. 21, no. 8, pp. 887-894, 2015.

[15] N. R. Sundaresan, P. Vasudevan, L. Zhong et al., "The sirtuin SIRT6 blocks IGF-Akt signaling and development of cardiac hypertrophy by targeting c-Jun," Nature medicine, vol. 18, no. 11, pp. 1643-1650, 2012.

[16] S. A. Trammell and C. Brenner, "NNMT: a bad actor in fat makes good in liver," Cell metabolism, vol. 22, no. 2, pp. 200201, 2015.

[17] A. Bürkle, "Physiology and pathophysiology of poly(ADPribosyl)ation," BioEssays, vol. 23, no. 9, pp. 795-806, 2001.

[18] J. Saldeen and N. Welsh, "Nicotinamide-induced apoptosis in insulin producing cells is associated with cleavage of poly(ADP-ribose) polymerase," Molecular and Cellular Endocrinology, vol. 139, no. 1-2, pp. 99-107, 1998.

[19] F. Li, Z. Z. Chong, and K. Maiese, "Cell Life versus cell longevity: the mysteries surrounding the $\mathrm{NAD}^{+}$precursor nicotinamide," Current medicinal chemistry, vol. 13, no. 8, pp. 883-895, 2006.

[20] J. H. Li and Z. H. Wang, "Association between urinary lowmolecular-weight metabolites and body mass index," International Journal of Obesity, vol. 35, no. S2, p. 554, 2011.

[21] R. M. Salek, M. L. Maguire, E. Bentley et al., "A metabolomic comparison of urinary changes in type 2 diabetes in mouse, rat, and human," Physiological Genomics, vol. 29, no. 2, pp. 99-108, 2007.

[22] M. Liu, L. Li, J. Chu et al., "Serum N(1)-methylnicotinamide is associated with obesity and diabetes in Chinese," The Journal of Clinical Endocrinology \& Metabolism, vol. 100, no. 8, pp. 3112-3117, 2015.

[23] C. Wu, C. Orozco, J. Boyer et al., "BioGPS: an extensible and customizable portal for querying and organizing gene annotation resources," Genome biology, vol. 10, no. 11, p. R130, 2009.

[24] J. Alexander, G. Q. Chang, J. T. Dourmashkin, and S. F. Leibowitz, "Distinct phenotypes of obesity-prone AKR/J, DBA2J and C57BL/6J mice compared to control strains," International Journal of Obesity, vol. 30, no. 1, pp. 50-59, 2006.

[25] K. L. Svenson, R. Von Smith, P. A. Magnani et al., "Multiple trait measurements in 43 inbred mouse strains capture the phenotypic diversity characteristic of human populations," Journal of applied physiology, vol. 102, no. 6, pp. 2369-2378, 2007.

[26] S. C. Grubb, T. P. Maddatu, C. J. Bult, and M. A. Bogue, "Mouse phenome database," Nucleic acids research, vol. 37, no. Database issue, pp. D720-D730, 2009.

[27] Y. H. Lee, S. Nair, E. Rousseau et al., "Microarray profiling of isolated abdominal subcutaneous adipocytes from obese vs non-obese Pima Indians: increased expression of inflammation-related genes," Diabetologia, vol. 48, no. 9, pp. 1776-1783, 2005. 
[28] S. Brachs, J. Polack, M. Brachs et al., "Genetic nicotinamide Nmethyltransferase (Nnmt) deficiency in male mice improves insulin sensitivity in diet-induced obesity but does not affect glucose tolerance,” Diabetes, vol. 68, no. 3, pp. 527-542, 2019.

[29] Q. Zhou, X. J. Zhu, and J. H. Li, “Association between nicotinamide N-methyltransferase gene polymorphisms and obesity in Chinese Han male college students," BioMed Research International, vol. 2017, Article ID 2984826, 6 pages, 2017.

[30] X. J. Zhu, Y. J. Lin, W. Chen et al., "Physiological study on association between nicotinamide $\mathrm{N}$-methyltransferase gene polymorphisms and hyperlipidemia," BioMed Research International, vol. 2016, Article ID 7521942, 8 pages, 2016.

[31] M. Bañales-Luna, N. Figueroa-Vega, C. I. Marín-Aragón et al., "Associations of nicotidamide-N-methyltransferase, FTO, and IRX3 genetic variants with body mass index and resting energy expenditure in Mexican subjects," Scientific Reports, vol. 10, no. 1, p. 11478, 2020.

[32] H. Yaguchi, K. Togawa, M. Moritani, and M. Itakura, "Identification of candidate genes in the type 2 diabetes modifier locus using expression QTL," Genomics, vol. 85, no. 5, pp. 591-599, 2005.

[33] A. Kannt, A. Pfenninger, L. Teichert et al., "Association of nicotinamide- $\mathrm{N}$-methyltransferase mRNA expression in human adipose tissue and the plasma concentration of its product, 1-methylnicotinamide, with insulin resistance," Diabetologia, vol. 58, no. 4, pp. 799-808, 2015.

[34] J. H. Li, Y. H. Wang, X. J. Zhu, Q. Zhou, Z. H. Xie, and T. F. Yao, "Metabolomics study on the association between nicotinamide N-methyltransferase gene polymorphisms and type 2 diabetes," International Journal of Diabetes in Developing Countries, vol. 38, pp. 409-416, 2018.

[35] J. C. Souto, F. Blanco-Vaca, J. M. Soria et al., “A genomewide exploration suggests a new candidate gene at chromosome $11 \mathrm{q} 23$ as the major determinant of plasma homocysteine levels: results from the GAIT project," The American Journal of Human Genetics, vol. 76, no. 6, pp. 925-933, 2005.

[36] A. Sazci, M. D. Ozel, E. Ergul, and C. Aygun, "Association of nicotinamide-N-methyltransferase gene rs694539 variant with patients with nonalcoholic steatohepatitis," Genetic Testing and Molecular Biomarkers, vol. 17, no. 11, pp. 849-853, 2013.

[37] L. M. van Driel, H. P. Smedts, W. A. Helbing et al., "Eight-fold increased risk for congenital heart defects in children carrying the nicotinamide $\mathrm{N}$-methyltransferase polymorphism and exposed to medicines and low nicotinamide," European Heart Journal, vol. 29, no. 11, pp. 1424-1431, 2008.

[38] A. Sazci, G. Sazci, B. Sazci, E. Ergul, and H. A. Idrisoglu, "Nicotinamide-N-methyltransferase gene rs694539 variant and migraine risk," The journal of headache and pain, vol. 17, no. 1, p. 93, 2016.

[39] A. Sazci, M. D. Ozel, E. Ergul, and M. E. Onder, “Association of nicotinamide-N-methyltransferase (NNMT) gene rs694539 variant with bipolar disorder," Gene, vol. 532, no. 2, pp. 272-275, 2013.

[40] A. Bromberg, E. Lerer, M. Udawela et al., "Nicotinamide-Nmethyltransferase (NNMT) in schizophrenia: genetic association and decreased frontal cortex mRNA levels," The International Journal of Neuropsychopharmacology, vol. 15, no. 6, pp. 727-737, 2012.

[41] G. Sazci, B. Sazci, A. Sazci, and H. A. Idrisoglu, "Association of nicotinamide-N-methyltransferase gene rs694539 variant with epilepsy," Molecular Neurobiology, vol. 53, no. 6, pp. 41974200, 2016.
[42] B. Giusti, C. Saracini, P. Bolli et al., "Genetic analysis of 56 polymorphisms in 17 genes involved in methionine metabolism in patients with abdominal aortic aneurysm," Journal of Medical Genetics, vol. 45, no. 11, pp. 721-730, 2008.

[43] R. B. Goldberg and K. Mather, "Targeting the consequences of the metabolic syndrome in the Diabetes Prevention Program," Arteriosclerosis, thrombosis, and vascular biology, vol. 32, no. 9, pp. 2077-2090, 2012.

[44] P. Belenky, K. L. Bogan, and C. Brenner, "NAD+ metabolism in health and disease," Trends in biochemical sciences, vol. 32, no. 1, pp. 12-19, 2007, Epub 2006 Dec 11. Erratum in: Trends Biochem Sci. 2008 Jan; 33(1): 1.

[45] J. H. Li, W. Chen, X. J. Zhu et al., "Associations of nicotinamide $\mathrm{N}$-methyltransferase gene single nucleotide polymorphisms with sport performance and relative maximal oxygen uptake," Journal of Sports Sciences, vol. 35, no. 22, pp. 21852190, 2017.

[46] R. H. Houtkooper, C. Cantó, R. J. Wanders, and J. Auwerx, "The secret life of NAD+: an old metabolite controlling new metabolic signaling pathways," Endocrine reviews, vol. 31, no. 2, pp. 194-223, 2010.

[47] R. Teperino, K. Schoonjans, and J. Auwerx, "Histone methyl transferases and demethylases; can they link metabolism and transcription?," Cell metabolism, vol. 12, no. 4, pp. 321-327, 2010.

[48] J. Jell, S. Merali, M. L. Hensen et al., "Genetically altered expression of spermidine/spermine N1-acetyltransferase affects fat metabolism in mice via acetyl-CoA," The Journal of Biological Chemistry, vol. 282, no. 11, pp. 8404-8413, 2007.

[49] J. G. Doench, C. P. Petersen, and P. A. Sharp, "siRNAs can function as miRNAs," Genes \& development, vol. 17, no. 4, pp. 438-442, 2003.

[50] A. L. Jackson, S. R. Bartz, J. Schelter et al., "Expression profiling reveals off-target gene regulation by RNAi," Nature Biotechnology, vol. 21, no. 6, pp. 635-637, 2003, Epub 2003 May 18.

[51] S. P. Persengiev, X. Zhu, and M. R. Green, "Nonspecific, concentration-dependent stimulation and repression of mammalian gene expression by small interfering RNAs (siRNAs)," $R N A$, vol. 10, no. 1, pp. 12-18, 2004.

[52] P. C. Scacheri, O. Rozenblatt-Rosen, N. J. Caplen et al., "Short interfering RNAs can induce unexpected and divergent changes in the levels of untargeted proteins in mammalian cells," Proceedings of the National Academy of Sciences, vol. 101, no. 7, pp. 1892-1897, 2004.

[53] A. L. Jackson, J. Burchard, J. Schelter et al., "Widespread siRNA "off-target" transcript silencing mediated by seed region sequence complementarity," RNA, vol. 12, no. 7, pp. 1179$1187,2006$.

[54] D. Grimm, K. L. Streetz, C. L. Jopling et al., "Fatality in mice due to oversaturation of cellular microRNA/short hairpin RNA pathways,” Nature, vol. 441, no. 7092, pp. 537-541, 2006.

[55] T. C. Roberts, R. Langer, and M. J. A. Wood, "Advances in oligonucleotide drug delivery," Nature Reviews Drug Discovery, vol. 19, no. 10, pp. 673-694, 2020.

[56] H. Neelakantan, H. Y. Wang, V. Vance, J. D. Hommel, S. F. McHardy, and S. J. Watowich, "Structure-activity relationship for small molecule inhibitors of nicotinamide N-methyltransferase," Journal of Medicinal Chemistry, vol. 60, no. 12, pp. 5015-5028, 2017.

[57] M. J. van Haren, R. Taig, J. Kuppens et al., "Inhibitors of nicotinamide $\mathrm{N}$-methyltransferase designed to mimic the 
methylation reaction transition state," Organic \& Biomolecular Chemistry, vol. 15, no. 31, pp. 6656-6667, 2017.

[58] A. Kannt, S. Rajagopal, S. V. Kadnur et al., "A small molecule inhibitor of nicotinamide N-methyltransferase for the treatment of metabolic disorders," Scientific reports, vol. 8, no. 1, p. 3660, 2018.

[59] N. Babault, A. Allali-Hassani, F. Li et al., "Discovery of bisubstrate inhibitors of nicotinamide $\mathrm{N}$-methyltransferase (NNMT)," Journal of medicinal chemistry, vol. 61, no. 4, pp. 1541-1551, 2018, Epub 2018 Jan 31. Erratum in: J Med Chem. 2018 Jul 12; 61(13): 5771-5772.

[60] Y. Gao, M. J. van Haren, E. E. Moret et al., "Bisubstrate inhibitors of nicotinamide N-methyltransferase (NNMT) with enhanced activity," Journal of medicinal chemistry, vol. 62, no. 14, pp. 6597-6614, 2019.

[61] H. Y. Lee, R. M. Suciu, B. D. Horning, E. V. Vinogradova, O. A. Ulanovskaya, and B. F. Cravatt, "Covalent inhibitors of nicotinamide N-methyltransferase (NNMT) provide evidence for target engagement challenges in situ," Bioorganic \& Medicinal Chemistry Letters, vol. 28, no. 16, pp. 2682-2687, 2018, Epub 2018 Apr 10.

[62] S. Ruf, M. S. Hallur, N. K. Anchan et al., "Novel nicotinamide analog as inhibitor of nicotinamide N-methyltransferase," Bioorganic \& medicinal chemistry letters, vol. 28, no. 5, pp. 922-925, 2018, Epub 2018 Jan 31.

[63] K. Przyborowski, M. Wojewoda, B. Sitek et al., "Effects of 1methylnicotinamide (MNA) on exercise capacity and endothelial response in diabetic mice," PLoS One, vol. 10, no. 6, article e0130908, 2015.

[64] S. Swaminathan, S. Birudukota, M. K. Thakur et al., "Crystal structures of monkey and mouse nicotinamide $\mathrm{N}$ methyltransferase (NNMT) bound with end product, 1methyl nicotinamide," Biochemical and Biophysical Research Communications, vol. 491, no. 2, pp. 416-422, 2017, Epub 2017 Jul 15.

[65] R. M. Menon, M. A. González, M. H. Adams, D. S. Tolbert, J. H. Leu, and E. A. Cefali, "Effect of the rate of niacin administration on the plasma and urine pharmacokinetics of niacin and its metabolites," Journal of Clinical Pharmacology, vol. 47, no. 6, pp. 681-688, 2007, Epub 2007 Apr 26.

[66] K. Shibata, T. Kawada, and K. Iwai, "Microdetermination of N1-methyl-2-pyridone-5-carboxamide, a major metabolite of nicotinic acid and nicotinamide, in urine by highperformance liquid chromatography," Journal of Chromatography, vol. 417, no. 1, pp. 173-177, 1987.

[67] K. Shibata, T. Kawada, and K. Iwai, "Simultaneous microdetermination of nicotinamide and its major metabolites, N1-methyl-2-pyridone-5-carboxamide and N1-methyl-4-pyridone-3-carboxamide, by high-performance liquid chromatography," Journal of Chromatography, vol. 424, no. 1, pp. 23-28, 1988.

[68] J. M. Markert, C. M. Fuller, G. Y. Gillespie et al., "Differential gene expression profiling in human brain tumors," Physiological Genomics, vol. 5, no. 1, pp. 21-33, 2001.

[69] M. Tomida, I. Mikami, S. Takeuchi, H. Nishimura, and H. Akiyama, "Serum levels of nicotinamide N-methyltransferase in patients with lung cancer," Journal of Cancer Research and Clinical Oncology, vol. 135, no. 9, pp. 1223-1229, 2009, Epub 2009 Feb 26

[70] J. Kim, S. J. Hong, E. K. Lim et al., "Expression of nicotinamide $\mathrm{N}$-methyltransferase in hepatocellular carcinoma is associated with poor prognosis," Journal of Experimental \& Clinical Cancer Research, vol. 28, no. 1, p. 20, 2009.

[71] M. Yao, H. Tabuchi, Y. Nagashima et al., "Gene expression analysis of renal carcinoma: adipose differentiation-related protein as a potential diagnostic and prognostic biomarker for clear-cell renal carcinoma," The Journal of Pathology, vol. 205, no. 3, pp. 377-387, 2005.

[72] S. W. Tang, T. C. Yang, W. C. Lin et al., "Nicotinamide Nmethyltransferase induces cellular invasion through activating matrix metalloproteinase- 2 expression in clear cell renal cell carcinoma cells," Carcinogenesis, vol. 32, no. 2, pp. 138-145, 2011, Epub 2010 Nov 2.

[73] Y. Wu, M. S. Siadaty, M. E. Berens, G. M. Hampton, and D. Theodorescu, "Overlapping gene expression profiles of cell migration and tumor invasion in human bladder cancer identify metallothionein $1 \mathrm{E}$ and nicotinamide $\mathrm{N}$-methyltransferase as novel regulators of cell migration," Oncogene, vol. 27, no. 52, pp. 6679-6689, 2008.

[74] J. S. Jang, H. Y. Cho, Y. J. Lee, W. S. Ha, and H. W. Kim, “The differential proteome profile of stomach cancer: identification of the biomarker candidates," Oncology Research, vol. 14, no. 10, pp. 491-499, 2004.

[75] B. H. Lim, B. I. Cho, Y. N. Kim, J. W. Kim, S. T. Park, and C. W. Lee, "Overexpression of nicotinamide Nmethyltransferase in gastric cancer tissues and its potential post-translational modification," Experimental \& Molecular Medicine, vol. 38, no. 5, pp. 455-465, 2006.

[76] T. Yu, Y. T. Wang, P. Chen et al., "Effects of nicotinamide Nmethyltransferase on PANC-1 cells proliferation, metastatic potential and survival under metabolic stress," Cellular Physiology and Biochemistry, vol. 35, no. 2, pp. 710-721, 2015, Epub 2015 Jan 28.

[77] M. Roessler, W. Rollinger, S. Palme et al., "Identification of nicotinamide $\mathrm{N}$-methyltransferase as a novel serum tumor marker for colorectal cancer," Clinical Cancer Research, vol. 11, no. 18, pp. 6550-6557, 2005.

[78] D. Sartini, A. Santarelli, V. Rossi et al., "Nicotinamide Nmethyltransferase upregulation inversely correlates with lymph node metastasis in oral squamous cell carcinoma," Molecular medicine, vol. 13, no. 7-8, pp. 415-421, 2007.

[79] V. Pozzi, D. Sartini, S. Morganti et al., "RNA-mediated gene silencing of nicotinamide N-methyltransferase is associated with decreased tumorigenicity in human oral carcinoma cells," PLoS One, vol. 8, no. 8, article e71272, 2013.

[80] J. H. Li, L. Q. Qiu, X. J. Zhu, and C. X. Cai, "Influence of exercises using different energy metabolism systems on NNMT expression in different types of skeletal muscle fibers," Science \& Sports, vol. 32, pp. 27-32, 2017.

[81] Q. Zhou, Z. G. Huang, X. J. Zhu et al., "Effects of nicotinamide $\mathrm{N}$-methyltransferase (NNMT) inhibition on the aerobic and the anaerobic endurance exercise capacity," Science \& Sports, vol. 33, pp. e159-e165, 2018. 